УДК 517.9

\author{
M. M. Bokalo, O. V. Ilnytska
}

\title{
FOURIER PROBLEM FOR PARABOLIC EQUATIONS WITH VARIABLE EXPONENTS OF NONLINEARITY AND TIME DELAY
}

\begin{abstract}
M. M. Bokalo, O. V. Ilnytska. Fourier problems for parabolic equations with variable exponents of nonlinearity and time delay, Mat. Stud. 47 (2017), 47-58.

The Fourier problem for nonlinear parabolic equations with variable exponents of nonlinearity and time delay is considered. The existence and uniqueness of weak solutions of the problem are investigated. Also, its a priori estimates are obtained.
\end{abstract}

Introduction. In this paper we consider the Fourier problems for the nonlinear parabolic equations with variable exponents of nonlinearity and time depended delay. A typical example of the equations being studied here is

$$
u_{t}-\sum_{i=1}^{n}\left(\widehat{a}_{i}(x, t)\left|u_{x_{i}}\right|^{p_{i}(x)-2} u_{x_{i}}\right)_{x_{i}}+\widehat{a}_{0}(x, t)|u|^{p_{0}(x)-2} u+\int_{t-\tau(t)}^{t} \widehat{c}(x, t, s) u(x, s) d s=f(x, t),
$$

$(x, t) \in Q:=\Omega \times(-\infty, 0)$, where $\Omega$ is a bounded domain in $\mathbb{R}^{n}(n \in \mathbb{N}), \widehat{a}_{0}, \ldots, \widehat{a}_{n}$ are measurable positive functions on $Q, p_{0}, \ldots, p_{n}$ (the exponents of nonlinearity) are measurable bounded functions such that $p_{0}(x)>2, \ldots, p_{n}(x)>2$ for a.e. $x \in \Omega, \tau$ is a nonnegative continuous function, $\widehat{c}$ is a measurable bounded function, $f$ is an integrable function, $u$ is unknown function.

Fourier problems for evolution equations arise in modeling different nonstationary processes in nature, that started a long time ago and initial conditions do not affect on them in the actual time moment, but boundary conditions do affect them. Thus, we can assume that the initial time is $-\infty$, while 0 is the final time, and initial conditions can be replaced with the behaviour of the solution as time variable turns to $-\infty$. Fourier problem for evolution equations appears during modeling in many fields of science such as ecology, economics, physics, cybernetics, etc. (see, e.g., [6]-[8], [12]-[14], [30], [31], [33], [36]-[38]), and they have been widely studied. Good reference overview on problems without initial conditions for evolution equations without time delay can be found in [12].

Equation (1) in the case $Q=\Omega \times(0, T)$ or $Q=\Omega \times(-\infty, 0)$, where $\Omega$ is a domain in $\mathbb{R}^{n}, T>0$, is an example of nonlinear parabolic equations with variable exponents of nonlinearity. These equations describe many physical processes such as electromagnetic fields, electrorheological fluids, image reconstruction processes, current flow in variable temperature

2010 Mathematics Subject Classification: 35B45, 35D30, 35K55, 35R10.

Keywords: Nonlinear parabolic equation; equation with time delay; Fourier problem; anisotropic parabolic equation; equation with variable exponents of nonlinearity.

doi:10.15330/ms.47.1.47-58

(C) M. M. Bokalo, O. V. Ilnytska, 2017 
field (see [27], [35]). For investigation of such equations generalized Lebesgue and Sobolev spaces are used. The mentioned spaces were firstly introduced in [32]. Properties of these spaces were studied in [32], [22], [26], [28], [18] e.a. The various problems for nonlinear differential equations of type (1) with $\widehat{c}=0$ are actively being investigated (see, e.g., [27], [1], [2], [3] [6], [9], [13], [23], [29], and references therein).

Equations with time delay arise during modeling in population dynamics, in non-Newtonian filtration, heat flux, etc. ([24]). The equations of type (1) on finite time interval with both constant exponents of nonlinearity and time delay are studied in [4], [5], [25], [21], [17] and others. Good reference overview on such papers can be found in [25]. Note that in these papers the semigroups theory is used.

Partial differential equations with a variable delay are less studied, and we known only paper [11] and works of Chueshov and Rezounenko (in particular, [15], [34]), where equations of type (1) on finite time interval, with $p_{0}(x)=\ldots=p_{n}(x)=2, \tau=\tau(u)$, are considered. In [15], a certain abstract parabolic problem with the state dependent delay term of a rather general structure is investigated. In [34], a nonlinear partial functional differential equations with main linear elliptic operator and the non-local nonlinear term with delay is investigated. In [11] equations of type (1) on finite time interval with variable exponents of nonlinearity and time delay are investigated. For proving existence of solutions of problems considered in these papers the Galerkin approximation is used. Fourier problems for parabolic equations with constant time delay were investigated in [10], [19].

To the best of our knowledge, the Fourier problems for parabolic equations with variable exponents of nonlinearity and time depended delay are an untreated topic in the literature. These problems are considered in our paper. Existence and uniqueness of solution of the problem are proved. This paper can be considered as continuation of [11].

The paper is organized as follows. In Section 1, the main notations and functional spaces are introduced. The statement of the problem and formulation of the main result are given in Section 2. The main result is proved in Section 3.

1. Notation and auxiliary facts. Let $n$ be a natural number, $\mathbb{R}^{n}$ be the standard linear space of ordered collections $x=\left(x_{1}, \ldots, x_{n}\right)$ of real numbers with the norm $|x|:=\left(\left|x_{1}\right|^{2}+\ldots+\right.$ $\left.\left|x_{n}\right|^{2}\right)^{1 / 2}$. Suppose that $\Omega \subset \mathbb{R}^{n}$ is a bounded domain with the piecewise smooth boundary $\partial \Omega, \partial \Omega=\Gamma_{0} \cup \Gamma_{1}$, where $\Gamma_{0}$ is the closure of an open set on $\partial \Omega$ (in particular, either $\Gamma_{0}=\varnothing$ or $\left.\Gamma_{0}=\partial \Omega\right), \Gamma_{1}:=\partial \Omega \backslash \Gamma_{0}, \nu=\left(\nu_{1}, \ldots, \nu_{n}\right)$ is the unit outward pointing normal vector on the $\partial \Omega$. Denote $S:=(-\infty, 0], Q:=\Omega \times S, \Sigma_{0}:=\Gamma_{0} \times S, \Sigma_{1}:=\Gamma_{1} \times S, Q_{t_{1}, t_{2}}:=\Omega \times\left(t_{1}, t_{2}\right)$ for arbitrary real $t_{1}$ and $t_{2}, t_{1}<t_{2}$.

Let us introduce some functional spaces. Let either $G=\Omega$ or $G=Q_{t_{1}, t_{2}}\left(-\infty<t_{1}<\right.$ $\left.t_{2}<+\infty\right)$, or $G=Q$. Suppose that $r \in L_{\infty}(\Omega), r(x) \geq 1$ for a.e. $x \in \Omega$. Consider the linear space $L_{r(\cdot)}(G)$ of the measurable functions $v: G \rightarrow \mathbb{R}$ such that $\rho_{G, r}(v)<\infty$, where $\rho_{G, r}(v):=\int_{\Omega}|v(x)|^{r(x)} d x$ if $G=\Omega, \rho_{G, r}(v):=\iint_{Q_{t_{1}, t_{2}}}|v(x, t)|^{r(x)} d x d t$ if $G=Q_{t_{1}, t_{2}}(-\infty<$ $\left.t_{1}<t_{2}<+\infty\right)$, and $\rho_{G, r}(v):=\iint_{Q}|v(x, t)|^{r(x)} d x d t$ if $G=Q$. This is a Banach space with respect to the norm $\|v\|_{L_{r(\cdot)}(G)}:=\inf \left\{\lambda>0 \mid \rho_{G, r}(v / \lambda) \leq 1\right\}$ (see [26, p. 599]) and it is called a generalized Lebesgue space. Note that if $r(x)=r_{0}=$ const $\geq 1$ for a.e. $x \in \Omega$ then $\|\cdot\|_{L_{r(\cdot)}(G)}$ is equal to the standard norm $\|\cdot\|_{L_{r_{0}}(G)}$ of the Lebesgue space $L_{r_{0}}(G)$. Note also that the set $C(\bar{G})$ is dense in $L_{r(\cdot)}(G)$ (see [26, p. 603]). According to [26, p. 599], if $\underset{x \in \Omega}{\operatorname{ess} \inf } r(x)>1$, then the space $L_{r(\cdot)}(G)$ is reflexive and the conjugate space $\left[L_{r(\cdot)}(G)\right]^{\prime}$ equals $L_{r^{\prime}(\cdot)}(G)$, where the function $r^{\prime}$ is defined by the equality $\frac{1}{r(x)}+\frac{1}{r^{\prime}(x)}=1$ for a.e. $x \in \Omega$. However, there 
exist properties of standard Lebesgue spaces that is not valid for the generalized Lebesgue spaces. Thus the properties of the generalized Lebesgue spaces are not simple corollaries of the correspondent properties of the standard Lebesgue spaces (see, e.g., the mentioned papers).

We denote by $L_{r(\cdot), \text { loc }}(\bar{Q})$ the space of measurable functions $g: Q \rightarrow \mathbb{R}$ such that the restriction of $g$ on $Q_{t_{1}, t_{2}}$ belongs to $L_{r(\cdot)}\left(Q_{t_{1}, t_{2}}\right)$ for each $t_{1}, t_{2} \in S$. This space is complete locally convex with respect to the family of seminorms $\left\{\|\cdot\|_{L_{r(\cdot)}\left(Q_{t_{1}, t_{2}}\right)} \mid t_{1}, t_{2} \in S\right\}$. A sequence $\left\{g_{m}\right\}$ is said to be convergent strongly (resp., weakly) in $L_{r(\cdot), \text { loc }}(\bar{Q})$ provided the sequences of restrictions $\left\{\left.g_{m}\right|_{Q_{t_{1}, t_{2}}}\right\}$ are convergent strongly (resp., weakly) in $L_{r(\cdot)}\left(Q_{t_{1}, t_{2}}\right)$ for all $t_{1}, t_{2} \in S$. Similarly we can define the space $L_{\infty, \text { loc }}(\bar{Q})$.

Consider the function $p=\left(p_{0}, p_{1}, \ldots, p_{n}\right): \Omega \rightarrow \mathbb{R}^{n+1}$ such that following condition is satisfied:

$(\mathcal{P})$ for every $i \in\{0,1, \ldots, n\}, p_{i}: \Omega \rightarrow \mathbb{R}$ is a measurable function such that

$$
p_{i}^{-}:=\underset{x \in \Omega}{\operatorname{ess} \inf } p_{i}(x) \geq 2, \quad p_{i}^{+}:=\operatorname{ess~sup}_{x \in \Omega} p_{i}(x)<+\infty .
$$

Denote by $p^{\prime}=\left(p_{0}^{\prime}, \ldots, p_{n}^{\prime}\right): \Omega \rightarrow \mathbb{R}^{n+1}$ the vector-function such that $\frac{1}{p_{i}(x)}+\frac{1}{p_{i}^{\prime}(x)}=1$ for a.e. $x \in \Omega(i=\overline{0, n})$. Clearly that $p_{i}^{\prime}: \Omega \rightarrow \mathbb{R}$ is a measurable bounded function such that $\underset{x \in \Omega}{\operatorname{essinf}} p_{i}(x) \geq 1(i=\overline{0, n})$.

Now let us give the definitions of the following functional spaces. First, denote by $W_{p(\cdot)}^{1}(\Omega)$ the generalized Sobolev space of the functions $v \in L_{p_{0}(\cdot)}(\Omega)$ such that $v_{x_{i}} \in L_{p_{i}(\cdot)}(\Omega)$ $(i=\overline{1, n})$. This is a Banach space with respect to the norm $\|v\|_{W_{p(\cdot)}^{1}(\Omega)}:=\|v\|_{L_{p_{0}(\cdot)}(\Omega)}+$ $\sum_{i=1}^{n}\left\|v_{x_{i}}\right\|_{L_{p_{i}(\cdot)}(\Omega)}$. Let $\widetilde{W}_{p(\cdot)}^{1}(\Omega)$ be the subspace of $W_{p(\cdot)}^{1}(\Omega)$ that is the closure of the space $\widetilde{C}^{1}(\bar{\Omega}):=\left\{v \in C^{1}(\bar{\Omega})|v|_{\Gamma_{0}}=0\right\}$ in $W_{p(\cdot)}^{1}(\Omega)$. Set $V_{p}(\Omega):=\widetilde{W}_{p(\cdot)}^{1}(\Omega)$.

Next, for arbitrary $t_{1}, t_{2} \in \mathbb{R}$, we denote by $W_{p(\cdot)}^{1,0}\left(Q_{t_{1}, t_{2}}\right)$ the set of functions $w \in$ $L_{p_{0}(\cdot)}\left(Q_{t_{1}, t_{2}}\right)$ such that $w_{x_{i}} \in L_{p_{i}(\cdot)}\left(Q_{t_{1}, t_{2}}\right)$ for all $i \in\{1, \ldots, n\}$. We define the norm $\|w\|_{W_{p(\cdot)}^{1,0}\left(Q_{t_{1}, t_{2}}\right)}:=\|w\|_{L_{p_{0}(\cdot)}\left(Q_{t_{1}, t_{2}}\right)}+\sum_{i=1}^{n}\left\|w_{x_{i}}\right\|_{L_{p_{i}(\cdot)}\left(Q_{t_{1}, t_{2}}\right)}$. Denote by $\widetilde{W}_{p(\cdot)}^{1,0}\left(Q_{t_{1}, t_{2}}\right)$ the subspace of $W_{p(\cdot)}^{1,0}\left(Q_{t_{1}, t_{2}}\right)$ consisting of functions $v$ such that $v(\cdot, t) \in \widetilde{W}_{p(\cdot)}^{1}(\Omega)$ for a. e. $t \in\left[t_{1}, t_{2}\right]$.

Let us denote by $\widetilde{W}_{p(\cdot), \text { loc }}^{1,0}(\bar{Q})$ the linear space of measurable functions such that their restrictions on $Q_{t_{1}, t_{2}}$ belong to $\widetilde{W}_{p(\cdot)}^{1,0}\left(Q_{t_{1}, t_{2}}\right)$ for all $t_{1}, t_{2} \in S$. This space is complete locally convex with respect to the family of seminorms $\left\{\|\cdot\|_{W_{p(\cdot)}^{1,0}\left(Q_{t_{1}, t_{2}}\right)} \mid t_{1}, t_{2} \in \mathbb{R}\right\}$.

Denote by $F_{p^{\prime}(\cdot), \text { loc }}(Q)$ the space of vector-functions $\left(f_{0}, f_{1}, \ldots, f_{n}\right)$ such that for each $i \in\{0,1, \ldots, n\} f_{i} \in L_{p_{i}^{\prime}(\cdot), \text { loc }}(\bar{Q})(i=\overline{1, n})$, and for each $i \in\{1, \ldots, n\} f_{i}=0$ a.e. in some neighborhood of the surface $\Sigma_{1}$.

Finally, define by $C_{c}^{1}(I)$, where $I$ is an interval, a linear space defined on $I$ continuously differentiable finite functions, moreover, if $I=\left(t_{1}, t_{2}\right)$, then we will write $C_{c}^{1}\left(t_{1}, t_{2}\right)$ instead of $C_{c}^{1}\left(\left(t_{1}, t_{2}\right)\right)$.

The following auxiliary result, which had been proved in [9], will be used in the sequel.

Lemma 1. Suppose that $w \in \widetilde{W}_{p(\cdot)}^{1,0}\left(Q_{t_{1}, t_{2}}\right)$, where $t_{1}, t_{2} \in \mathbb{R}\left(t_{1}<t_{2}\right)$, satisfies the following identity

$$
\int_{t_{1}}^{t_{2}} \int_{\Omega}\left\{-w \psi \varphi^{\prime}+\left(g_{0} \psi+\sum_{i=1}^{n} g_{i} \psi_{x_{i}}\right) \varphi\right\} d x d t=0, \quad \psi \in V_{p}(\Omega), \varphi \in C_{c}^{1}\left(t_{1}, t_{2}\right),
$$


for some $g_{i} \in L_{p_{i}^{\prime}(\cdot)}\left(Q_{t_{1}, t_{2}}\right)(i=\overline{0, n})$.

Then $w \in C\left(\left[t_{1}, t_{2}\right] ; L_{2}(\Omega)\right)$ and for any $\sigma_{1}, \sigma_{2} \in\left[t_{1}, t_{2}\right]\left(\sigma_{1}<\sigma_{2}\right)$, for every $\theta \in C^{1}\left(\left[t_{1}, t_{2}\right]\right)$ we have

$$
\left.\frac{1}{2} \theta(t) \int_{\Omega}|w(x, t)|^{2} d x\right|_{t=\sigma_{1}} ^{t=\sigma_{2}}-\frac{1}{2} \int_{\sigma_{1}}^{\sigma_{2}} \int_{\Omega}|w|^{2} \theta^{\prime} d x d t+\int_{\sigma_{1}}^{\sigma_{2}} \int_{\Omega}\left\{g_{0} w+\sum_{i=1}^{n} g_{i} w_{x_{i}}\right\} \theta d x d t=0 .
$$

Remark 1. Note that from $w \in L_{2}(Q)$ it follows that

$$
\lim _{\sigma \rightarrow-\infty} \int_{\sigma-1}^{\sigma} \int_{\Omega}|w|^{2} d x d t=0
$$

If $w \in L_{2}(Q) \cap C\left(S, L_{2}(\Omega)\right)$, then it follows that there exists sequence $\left\{t_{k}\right\}_{k=0}^{\infty} \subset S$ such that $t_{k} \rightarrow-\infty$ as $k \rightarrow+\infty$, and

$$
\lim _{k \rightarrow+\infty} \int_{\Omega}\left|w\left(x, t_{k}\right)\right|^{2} d x=0
$$

2. Statement of the problem and main result. In this paper we consider the problem of finding a function $u: \bar{Q} \rightarrow \mathbb{R}$ satisfying (in some sense) the equation

$$
\begin{gathered}
u_{t}-\sum_{i=1}^{n} \frac{d}{d x_{i}} a_{i}(x, t, u, \nabla u)+a_{0}(x, t, u, \nabla u)+\int_{t-\tau(t)}^{t} c(x, t, s, u(x, s)) d s \\
=-\sum_{i=1}^{n} \frac{\partial}{\partial x_{i}} f_{i}(x, t)+f_{0}(x, t), \quad(x, t) \in Q,
\end{gathered}
$$

and the boundary conditions

$$
\left.u\right|_{\Sigma_{0}}=0,\left.\quad \sum_{i=1}^{n} a_{i}(x, t, u, \nabla u) \nu_{i}\right|_{\Sigma_{1}}=0
$$

Here $\tau: S \rightarrow \mathbb{R}$ is a continuous bounded function such that $\tau(t) \geq 0$ for all $t \in S, \tau^{+}:=$ $\sup \tau(t)$, and $a_{i}: Q \times \mathbb{R}^{1+n} \rightarrow \mathbb{R}, c: Q \times S \times \mathbb{R} \rightarrow \mathbb{R}, f_{i}: Q \rightarrow \mathbb{R}(i=\overline{0, n})$ are given $t \in S$
real-valued functions.

We introduce the following classes of the initial data.

Let $p$ satisfy condition $(\mathcal{P})$. Define $\mathcal{A}(p)$ to be the set of the collections $\left(a_{0}, a_{1}, \ldots, a_{n}\right)$ of the real-value functions $a_{i}: Q \times \mathbb{R}^{1+n} \rightarrow \mathbb{R}(i=\overline{0, n})$ satisfying the following assumptions:

$\left(\mathcal{A}_{1}\right)$ for every $i \in\{0,1, \ldots, n\}, a_{i}$ is the Caratheodory function (i.e., $a_{i}(x, t, \cdot, \cdot): \mathbb{R}^{1+n} \rightarrow$ $\mathbb{R}$ is a continuous for a.e. $(x, t) \in Q$, and $a_{i}(\cdot, \cdot, \rho, \xi): Q \rightarrow \mathbb{R}$ is a measurable for every $\left.(\rho, \xi) \in \mathbb{R}^{1+n}\right)$, and $a_{i}(x, t, 0,0)=0$ for a.e. $(x, t) \in Q$

$\left(\mathcal{A}_{2}\right)$ for every $i \in\{0,1, \ldots, n\}$, for a.e. $(x, t) \in Q$, and for every $(\rho, \xi) \in \mathbb{R}^{1+n}$ the following estimate is valid

$$
\left|a_{i}(x, t, \rho, \xi)\right| \leq C_{1}\left(\sum_{j=1}^{n}\left|\xi_{j}\right|^{p_{j}(x) / p_{i}^{\prime}(x)}+|\rho|^{p_{0}(x) / p_{i}^{\prime}(x)}\right)+h_{i}(x, t),
$$


where $C_{1}$ is a positive constant, and $h_{i} \in L_{p_{i}^{\prime}(\cdot)}(Q)$;

$\left(\mathcal{A}_{3}\right)$ for a.e. $(x, t) \in Q$ and for every $\left(\rho_{1}, \xi^{1}\right),\left(\rho_{2}, \xi^{2}\right) \in \mathbb{R}^{1+n}$ the following inequality holds

$$
\begin{aligned}
& \sum_{i=1}^{n}\left(a_{i}\left(x, t, \rho_{1}, \xi^{1}\right)\right.\left.-a_{i}\left(x, t, \rho_{2}, \xi^{2}\right)\right)\left(\xi_{i}^{1}-\xi_{i}^{2}\right)+\left(a_{0}\left(x, t, \rho_{1}, \xi^{1}\right)-a_{0}\left(x, t, \rho_{2}, \xi^{2}\right)\right)\left(\rho_{1}-\rho_{2}\right) \geq \\
& \geq K_{1}\left(\sum_{i=1}^{n}\left|\xi_{i}^{1}-\xi_{i}^{2}\right|^{p_{i}(x)}+\left|\rho_{1}-\rho_{2}\right|^{p_{0}(x)}\right)+K_{2}\left|\rho_{1}-\rho_{2}\right|^{2}
\end{aligned}
$$

where $K_{1}, K_{2}$ are positive constants.

Define $\mathcal{C}$ to be the set of the functions $c(x, t, s, \rho),(x, t, s, \rho) \in Q \times S \times \mathbb{R}$, satisfying the following assumptions:

$\left(\mathcal{C}_{1}\right) c$ is a Caratheodory function (i.e., $c(x, t, s, \cdot): \mathbb{R} \rightarrow \mathbb{R}$ is a continuous function for a.e. $(x, t, s) \in Q \times S$, and $c(\cdot, \cdot, \cdot, \rho): Q \times S \rightarrow \mathbb{R}$ is a measurable function for every $\rho \in \mathbb{R})$, in addition, $c(x, t, s, 0)=0$ for a.e. $(x, t, s) \in Q \times S$;

$\left(\mathcal{C}_{2}\right)$ there exists a constant $L>0$ (depending on $c$ ) such that for a.e. $(x, t, s) \in Q \times S$ and for every $\rho_{1}, \rho_{2} \in \mathbb{R}$ the inequality

$$
\left|c\left(x, t, s, \rho_{1}\right)-c\left(x, t, s, \rho_{2}\right)\right| \leq L\left|\rho_{1}-\rho_{2}\right|
$$

holds.

Remark 2. From the condition $c(x, t, s, 0)=0$ (see $\left.\left(\mathcal{C}_{1}\right)\right)$ and $\left(\mathcal{C}_{2}\right)$ follows that for a.e. $(x, t, s) \in Q \times S$, and for every $\rho \in \mathbb{R}$ the following estimate is valid

$$
|c(x, t, s, \rho)| \leq L|\rho| \text {. }
$$

Now we can give a definition of the weak solution of problem (6), (7).

Definition 1. Let $p$ satisfy condition $(\mathcal{P}),\left(a_{0}, a_{1}, \ldots, a_{n}\right) \in \mathcal{A}(p), c \in \mathcal{C},\left(f_{0}, f_{1}, \ldots, f_{n}\right) \in$ $F_{p^{\prime}(\cdot), \text { loc }}(\bar{Q})$. The function $u \in \widetilde{W}_{p(\cdot) \text { loc }}^{1,0}(\bar{Q}) \cap L_{2}(Q) \cap C\left(S ; L_{2}(\Omega)\right)$ is called a weak solution of problem (6), (7), if the integral equality

$$
\begin{gathered}
\iint_{Q}\left\{\sum_{i=1}^{n} a_{i}(x, t, u, \nabla u) v_{x_{i}} \varphi+a_{0}(x, t, u, \nabla u) v \varphi+v \varphi \int_{t-\tau(t)}^{t} c(x, t, s, u(x, s)) d s-\right. \\
\left.-u v \varphi^{\prime}\right\} d x d t=\iint_{Q}\left\{\sum_{i=1}^{n} f_{i} v_{x_{i}} \varphi+f_{0} v \varphi\right\} d x d t
\end{gathered}
$$

holds for every $v \in V_{p}(\Omega)$ and $\varphi \in C_{c}^{1}(-\infty, 0)$.

The main result of our paper is the following statement.

Theorem 1. Let $p$ satisfy condition $(\mathcal{P}),\left(a_{0}, a_{1}, \ldots, a_{n}\right) \in \mathcal{A}(p), c \in \mathcal{C},\left(f_{0}, f_{1}, \ldots, f_{n}\right) \in$ $L_{p_{0}(\cdot)}(Q) \times L_{p_{1}(\cdot)}(Q) \times \cdots \times L_{p_{n}(\cdot)}(Q)$, and

$$
K_{2}-2 L \tau^{+}>0
$$


Then problem (6), (7) has a unique weak solution, and it belongs to $\widetilde{W}_{p(\cdot)}^{1,0}(Q)$, and it satisfies the following estimate

$$
\begin{aligned}
\sup _{t \in S} \int_{\Omega}|u(x, t)|^{2} d x+ & \iint_{Q}\left\{\sum_{i=1}^{n}\left|u_{x_{i}}(x, t)\right|^{p_{i}(x)}+|u(x, t)|^{p_{0}(x)}+|u(x, t)|^{2}\right\} d x d t \leq \\
& \leq C_{2} \iint_{Q}\left\{\sum_{i=0}^{n}\left|f_{i}(x, t)\right|^{p_{i}^{\prime}(x)}\right\} d x d t,
\end{aligned}
$$

where $C_{2}>0$ is a constant depending only on $K_{1}, K_{2}, L, \tau^{+}$and $p_{i}^{-}(i=\overline{0, n})$.

3. Proof of the main result. For a function $w: Q \rightarrow \mathbb{R}$ we denote

$$
\begin{gathered}
a_{j}(w)(x, t):=a_{j}(x, t, w(x, t), \nabla w(x, t)), \quad(x, t) \in Q, \quad j=\overline{0, n}, \\
c(w)(x, t, s):=c(x, t, s, w(x, s)), \quad(x, t, s) \in Q \times S, \quad \partial_{i} w=w_{x_{i}}, \quad i=\overline{1, n}, \quad \partial_{0} w=w .
\end{gathered}
$$

Let us prove Theorem 1 in three steps: firstly, we prove the uniqueness of solution of problem (6), (7), later, its existence and, finally, we show correctness of estimate (13).

First step (uniqueness of solution). Assume the opposite. Let $u_{1}$ and $u_{2}$ be two different weak solutions of the problem. Denote $w:=u_{1}-u_{2}$. Consider the difference between (11) with $u=u_{2}$ and (11) with $u=u_{1}$, we obtain

$$
\begin{gathered}
-\iint_{Q} w v \varphi^{\prime} d x d t+\iint\left[\sum_{Q}^{n}\left(a_{i}\left(u_{1}\right)-a_{i}\left(u_{2}\right)\right) \partial_{i} v+\right. \\
\left.+v \int_{t-\tau(t)}^{t}\left(c\left(u_{1}\right)-c\left(u_{2}\right)\right) d s\right] \varphi d x d t=0 \quad \forall v \in V_{p}(\Omega), \quad \forall \varphi \in C_{c}^{1}(-\infty, 0) .
\end{gathered}
$$

According to Lemma 1 , with $\theta(t)=1, t \in \mathbb{R}$, from equality (14) we get

$$
\begin{gathered}
\frac{1}{2} \int_{\Omega}\left|w\left(x, \sigma_{2}\right)\right|^{2} d x-\frac{1}{2} \int_{\Omega}\left|w\left(x, \sigma_{1}\right)\right|^{2} d x+\int_{\sigma_{1}} \int_{\Omega}^{\sigma_{2}}\left[\sum_{i=0}^{n}\left(a_{i}\left(u_{1}\right)-a_{i}\left(u_{2}\right)\right)\left(\partial_{i} u_{1}-\partial_{i} u_{2}\right)+\right. \\
\left.+\underset{t-\tau(t)}{w}\left(c\left(u_{1}\right)-c\left(u_{2}\right)\right) d s\right] d x d t=0,
\end{gathered}
$$

where $\sigma_{1}, \sigma_{2} \in S\left(\sigma_{1}<\sigma_{2}\right)$ are arbitrary.

From condition $\left(\mathcal{A}_{3}\right)$ for a.e. $(x, t) \in Q$ we have

$$
\sum_{i=0}^{n}\left(a_{i}\left(u_{1}\right)-a_{i}\left(u_{2}\right)\right)\left(\partial_{i} u_{1}-\partial_{i} u_{2}\right) \geq K_{1} \sum_{i=0}^{n}\left|\partial_{i} u_{1}-\partial_{i} u_{2}\right|^{p_{i}(x)}+K_{2}\left|u_{1}-u_{2}\right|^{2}
$$

Consider the last term from equality (15). Using condition $\left(\mathcal{C}_{2}\right)$, Fubini Theorem and 
Hölder's inequality, for a.e. $x \in \Omega$ we obtain

$$
\begin{gathered}
\left|\int_{\sigma_{1}}^{\sigma_{2}} w(x, t)\left(\int_{t-\tau(t)}^{t}\left(c\left(u_{1}\right)(x, t, s)-c\left(u_{2}\right)(x, t, s)\right) d s\right) d t\right| \leq L \int_{\sigma_{1}}^{\sigma_{2}}|w(x, t)|\left(\int_{t-\tau^{+}}^{t}|w(x, s)| d s\right) d t \leq \\
\leq L \sqrt{\tau^{+}}\left(\int_{\sigma_{1}}^{\sigma_{2}}|w(x, t)|^{2} d t\right)^{1 / 2}\left(\int_{\sigma_{1}}^{\sigma_{2}}\left(\int_{t-\tau^{+}}^{t}|w(x, s)|^{2} d s\right) d t\right)^{1 / 2} .
\end{gathered}
$$

Now consider the second integral in right-hand side of inequality (17). Changing order of integration and assuming $w(x, t)=0$ for $x \in \Omega, t>0$, for a.e. $x \in \Omega$ we have

$$
\int_{\sigma_{1}}^{\sigma_{2}}\left(\int_{t-\tau^{+}}^{t}|w(x, s)|^{2} d s\right) d t \leq \int_{\sigma_{1}-\tau^{+}}^{\sigma_{2}}|w(x, s)|^{2} d s \int_{s}^{s+\tau^{+}} d t=\tau^{+}\left(\int_{\sigma_{1}}^{\sigma_{2}}|w(x, s)|^{2} d s+\int_{\sigma_{1}-\tau^{+}}^{\sigma_{1}}|w(x, s)|^{2} d s\right) .
$$

Substituting (18) in (17), the last term from obtained above relations chain instead of the first one, and using Cauchy inequality: $\sqrt{a b} \leq a+b(a \geq 0, b \geq 0)$, we obtain

$$
\begin{gathered}
\left|\int_{\sigma_{1}}^{\sigma_{2}} w(x, t)\left(\int_{t-\tau(t)}^{t}\left(c\left(u_{1}\right)(x, t, s)-c\left(u_{1}\right)(x, t, s)\right) d s\right) d t\right| \leq \\
\quad \leq L \tau^{+}\left(2 \int_{\sigma_{1}}^{\sigma_{2}}|w(x, t)|^{2} d t+\int_{\sigma_{1}-\tau^{+}}^{\sigma_{1}}|w(x, t)|^{2} d t\right) .
\end{gathered}
$$

Using (16), (19), from (15) we obtain

$$
\begin{aligned}
\frac{1}{2} \int_{\Omega}\left|w\left(x, \sigma_{2}\right)\right|^{2} d x+ & K_{1} \int_{\sigma_{1}}^{\sigma_{2}} \int_{\Omega}\left(\sum_{i=0}^{n}\left|\partial_{i} u_{1}-\partial_{i} u_{2}\right|^{p_{i}(x)}\right) d x+\left(K_{2}-2 L \tau^{+}\right) \int_{\sigma_{1}}^{\sigma_{2}} \int_{\Omega}|w(x, t)|^{2} d x d t \leq \\
& \leq \frac{1}{2} \int_{\Omega}\left|w\left(x, \sigma_{1}\right)\right|^{2} d x+L \tau^{+} \int_{\sigma_{1}-\tau^{+}}^{\sigma_{1}} \int_{\Omega}|w(x, t)|^{2} d x d t .
\end{aligned}
$$

Then, taking into account (12), we have

$$
\int_{\Omega}\left|w\left(x, \sigma_{2}\right)\right|^{2} d x \leq \int_{\Omega}\left|w\left(x, \sigma_{1}\right)\right|^{2} d x+2 L \tau^{+} \int_{\sigma_{1}-\tau^{+}}^{\sigma_{1}} \int_{\Omega}|w(x, t)|^{2} d x d t .
$$

Since $w \in L_{2}(Q) \cap C\left(S, L_{2}(\Omega)\right)$, according to Remark 1 , there exists a sequence $\left\{\sigma_{1, k}\right\}_{k=0}^{\infty} \subset S$, such that $\sigma_{1, k} \underset{k \rightarrow+\infty}{\longrightarrow}-\infty$ and $\int_{\Omega}\left|w\left(x, \sigma_{1, k}\right)\right|^{2} d x+2 L \tau^{+} \int_{\sigma_{1, k}-\tau^{+}}^{\sigma_{1, k}} \int_{\Omega}|w(x, t)|^{2} d x d t \underset{k \rightarrow+\infty}{\rightarrow} 0$.

Let us fix an arbitrary $\sigma_{2}$ in (20), and take in (20) $\sigma_{1, k}$ for $k \in \mathbb{N}$ such that $\sigma_{1, k}<\sigma_{2}$ instead of $\sigma_{1}$. Tending $k$ to $+\infty$, we get the equality $\int_{\Omega}\left|w\left(x, \sigma_{2}\right)\right|^{2} d x=0$. Since $\sigma_{2} \in S$ is arbitrary, we obtain $w(x, t)=0$ for a.e. $(x, t) \in Q$, that contradicts to our assumption. Therefore, a weak solution of problem (6), (7) is unique. 
Second step (existence of solution). For each $m \in N$ denote $Q_{m}=\Omega \times(-m, 0], \tau_{m}=$ $\min _{-m \leq t \leq 0}\{t-\tau(t)\}$. It is clear that $\tau_{m} \leq-m$. Denote $f_{i, m}(\cdot, t):=\left\{\begin{array}{ll}f_{i}(\cdot, t), & -m<t \leq 0 ; \\ 0, & t \leq-m,\end{array}\right.$ and consider the problem: to find a function $u_{m} \in \widetilde{W}_{p(\cdot)}^{1,0}\left(Q_{m}\right) \cap C\left(\left[-\tau_{m}, 0\right] ; L_{2}(\Omega)\right)$, which satisfies the initial condition

$$
u_{m}(x, t)=0, \quad(x, t) \in \bar{\Omega} \times\left[\tau_{m},-m\right]
$$

(if $\tau_{m}=-m$ then $\left[\tau_{m},-m\right]:=\{-m\}$ ) and equation (6) in $Q_{m}$ in the sense of integral equality

$$
\begin{aligned}
& \iint_{Q_{m}}\left\{\sum_{i=1}^{n} a_{i}\left(x, t, u_{m}, \nabla u_{m}\right) v_{x_{i}} \varphi+a_{0}\left(x, t, u_{m}, \nabla u_{m}\right) v \varphi+v \varphi \int_{t-\tau(t)}^{t} c\left(x, t, s, u_{m}(x, s)\right) d s-\right. \\
& \left.-u_{m} v \varphi^{\prime}\right\} d x d t=\iint_{Q_{m}}\left\{\sum_{i=1}^{n} f_{i, m} v_{x_{i}} \varphi+f_{0, m} v \varphi\right\} d x d t, \quad v \in V_{p}, \quad \varphi \in C_{c}^{1}(-m, 0) .
\end{aligned}
$$

Existence and uniqueness of solution of this problem follows from results of the paper [11]. For each $m \in \mathbb{N}$ we extend $u_{m}$ by 0 on all $\Omega \times \mathbb{R}$ and denote this extension as $u_{m}$.

Now we shall get estimates of $u_{m}$ for each $m \in \mathbb{N}$. Firstly, note that for each $m \in \mathbb{N}$ function $u_{m}$ belongs to $\widetilde{W}_{p(\cdot)}^{1,0}(Q) \cap L_{2}(Q) \cap C\left(S ; L_{2}(\Omega)\right)$ and satisfies integral equality (11) with $f_{i, m}$ instead of $f_{i}$, i.e., the following equality is correct

$$
\begin{gathered}
\iint_{Q}\left\{\sum_{i=1}^{n} a_{i}\left(x, t, u_{m}, \nabla u_{m}\right) v_{x_{i}} \varphi+a_{0}\left(x, t, u_{m}, \nabla u_{m}\right) v \varphi+v \varphi \int_{t-\tau(t)}^{t} c\left(x, t, s, u_{m}(x, s)\right) d s-\right. \\
\left.-u_{m} v \varphi^{\prime}\right\} d x d t=\iint_{Q}\left\{\sum_{i=1}^{n} f_{i, m} v_{x_{i}} \varphi+f_{0, m} v \varphi\right\} d x d t, \quad v \in V_{p}(\Omega), \quad \varphi \in C_{c}^{1}(-\infty, 0) .
\end{gathered}
$$

Further we need Young's inequality in the form

$$
a b \leq \varepsilon|a|^{r(x)}+\varepsilon^{-\frac{1}{r^{-}-1}}|b|^{r^{\prime}(x)} \quad \text { for a.e. } x \in \Omega, \quad a, b \in \mathbb{R}, \quad 0<\varepsilon \leq 1,
$$

where $r \in L^{\infty}(\Omega), r(x)>1, r^{\prime}(x):=r(x) /(r(x)-1)$ for a.e. $x \in \Omega, r^{-}:=\underset{x \in \Omega}{\operatorname{ess} \inf } r(x)>1$.

Applying Lemma 1 , with $\theta(t)=2, \sigma_{1}, \sigma_{2} \subset S,\left(\sigma_{1}<\sigma_{2}\right)$ to equality (23), we obtain

$$
\begin{aligned}
& \int_{\Omega}\left|u_{m}\left(x, \sigma_{2}\right)\right|^{2} d x-\int_{\Omega}\left|u_{m}\left(x, \sigma_{1}\right)\right|^{2} d x+2 \int_{\sigma_{1}} \int_{\Omega}^{\sigma_{2}}\left[\sum_{i=0}^{n} a_{i}\left(u_{m}\right) \partial_{i} u_{m}\right] d x d t+ \\
& +2 \int_{\sigma_{1}}^{\sigma_{2}} \int_{\Omega} u_{m}\left[\int_{t-\tau(t)}^{t} c\left(u_{m}\right)(x, t, s) d s\right] d x d t=2 \int_{\sigma_{1}}^{\sigma_{2}} \int_{\Omega}\left\{\sum_{i=0}^{n} f_{i, m} \partial_{i} u_{m}\right\} d x d t .
\end{aligned}
$$

Applying inequality (24), the following estimation can be obtained

$$
\int_{\sigma_{1}}^{\sigma_{2}} \int_{\Omega}\left\{\sum_{i=0}^{n} f_{i, m} \partial_{i} u_{m}\right\} d x d t \leq \varepsilon \int_{\sigma_{1}}^{\sigma_{2}} \int_{\Omega}\left\{\sum_{i=0}^{n}\left|\partial_{i} u_{m}(x, t)\right|^{p_{i}(x)}\right\} d x d t+
$$




$$
+\int_{\sigma_{1}}^{\sigma_{2}} \int_{\Omega}\left\{\sum_{i=0}^{n} \varepsilon^{-\frac{1}{p_{i}^{-}-1}}\left|f_{i}(x, t)\right|^{p_{i}^{\prime}(x)}\right\} d x d t
$$

Analogically as inequality (19) has been obtained (using (10) instead of condition $\left(\mathcal{C}_{2}\right)$ ), we can get

$$
\left|\int_{\sigma_{1}}^{\sigma_{2}} u_{m}(x, t) \int_{t-\tau(t)}^{t} c\left(u_{m}\right)(x, t, s) d s d t\right| \leq L \tau^{+}\left(2 \int_{\sigma_{1}}^{\sigma_{2}}\left|u_{m}(x, t)\right|^{2} d t+\int_{\sigma_{1}-\tau^{+}}^{\sigma_{1}}\left|u_{m}(x, t)\right|^{2} d t\right) .
$$

From conditions $\left(\mathcal{A}_{1}\right),\left(\mathcal{A}_{3}\right)$ we have

$$
\int_{\sigma_{1}}^{\sigma_{2}} \int_{\Omega}\left[\sum_{i=0}^{n} a_{i}\left(u_{m}\right) \partial_{i} u_{m}\right] d x d t \geq \int_{\sigma_{1}}^{\sigma_{2}} \int_{\Omega}\left[K_{1} \sum_{i=0}^{n}\left|\partial_{i} u\right|^{p_{i}(x)}+K_{2}|u|^{2}\right] d x d t .
$$

From inequality (25), using estimates (26)-(28) and taking $\sigma_{1}<-m$, we obtain

$$
\begin{gathered}
\int_{\Omega}\left|u_{m}\left(x, \sigma_{2}\right)\right|^{2} d x+2\left(K_{1}-\varepsilon\right) \int_{\sigma_{1}} \int_{\Omega}\left\{\sum_{i=0}^{n}\left|\partial_{i} u_{m}(x, t)\right|^{p_{i}(x)}\right\} d x d t+ \\
+2\left(K_{2}-2 L \tau^{+}\right) \int_{\sigma_{1}} \int_{\Omega}^{\sigma_{2}}\left|u_{m}(x, t)\right|^{2} d x d t \leq 2 \int_{\sigma_{1}}^{\sigma_{2}} \int_{\Omega}\left\{\sum_{i=0}^{n} \varepsilon^{-\frac{1}{p_{i}^{-}-1}}\left|f_{i, m}(x, t)\right|^{p_{i}^{\prime}(x)}\right\} d x d t .
\end{gathered}
$$

Taking $\varepsilon=\frac{1}{2} \min \left\{K_{1}, 1\right\}$ and using (12), from (29) we have

$$
\begin{gathered}
\sup _{\sigma \in S} \int_{\Omega}\left|u_{m}(x, \sigma)\right|^{2} d x+C_{4} \iint_{Q}\left\{\sum_{i=0}^{n}\left|\partial_{i} u_{m}(x, t)\right|^{p_{i}(x)}+\left|u_{m}(x, t)\right|^{2}\right\} d x d t \leq \\
\leq C_{5} \iint_{Q}\left\{\sum_{i=0}^{n}\left|f_{i, m}(x, t)\right|^{p_{i}^{\prime}(x)}\right\} d x d t
\end{gathered}
$$

where $C_{4}$ and $C_{5}$ are positive constants depending only on $K_{1}, K_{2}, L, \tau^{+}$and $p_{i}^{-}(i=\overline{0, n})$.

According to definition of $f_{i, m}$ from (30) we have

$$
\begin{gathered}
\sup _{\sigma \in S} \int_{\Omega}\left|u_{m}(x, \sigma)\right|^{2} d x+\iint_{Q}\left\{\sum_{i=0}^{n}\left|\partial_{i} u_{m}(x, t)\right|^{p_{i}(x)}+\left|u_{m}(x, t)\right|^{2}\right\} d x d t \leq \\
\leq C_{6} \iint_{Q}\left\{\sum_{i=0}^{n}\left|f_{i}(x, t)\right|^{p_{i}^{\prime}(x)}\right\} d x d t
\end{gathered}
$$

where $C_{6}>0$ is a positive constant, depending only on $\tau^{+}, K_{1}, K_{2}, L$ and $p_{i}(i=\overline{0, n})$.

Let us show that $\left\{u_{m}\right\}$ is a Cauchy sequence. Taking arbitrary $k, l \in \mathbb{N}$ such that $k<l$ and considering difference between $u_{k}$ and $u_{l}$, similarly as estimate (30), for any $\sigma \in S$ such 
that $-k \leq \sigma \leq 0$ one can obtain

$$
\begin{gathered}
\int_{\Omega}\left|u_{k}(x, \sigma)-u_{l}(x, \sigma)\right|^{2} d x+\int_{-l}^{\sigma} \int_{\Omega}\left\{\sum_{i=0}^{n}\left|\partial_{i} u_{k}-\partial_{i} u_{l}\right|^{p_{i}(x)}+\left|u_{k}-u_{l}\right|^{2}\right\} d x d t \leq \\
\leq C_{7} \int_{-l}^{-k} \int_{\Omega} \sum_{i=0}^{n}\left|f_{i, k}(x, t)-f_{i, l}(x, t)\right|^{p_{i}^{\prime}(x)} d x d t,
\end{gathered}
$$

where $C_{7}$ is a positive constant independent of $k, l$.

The condition $f_{i} \in L_{p_{i}^{\prime}(\cdot)}(Q)(i=\overline{0, n})$ implies that the right-hand side of inequality (32) tends to zero when $k$ and $l$ tend to $+\infty$. This means that the sequence $\left\{u_{m}\right\}_{m=1}^{\infty}$ is a Cauchy sequence in the space $\widetilde{W}_{p(\cdot)}^{1,0}(Q) \cap L_{2}(Q) \cap C\left(S ; L_{2}(\Omega)\right)$. Consequently, there exists the function $u \in \widetilde{W}_{p(\cdot)}^{1,0}(Q) \cap L_{2}(Q) \cap C\left(S ; L_{2}(\Omega)\right)$ such that

$$
u_{m} \underset{m \rightarrow \infty}{\longrightarrow} u \quad \text { strongly in } \quad \widetilde{W}_{p(\cdot)}^{1,0}(Q) \cap L_{2}(Q) \cap C\left(S ; L_{2}(\Omega)\right) .
$$

From condition $\left(\mathcal{C}_{2}\right)$, Fubini Theorem, Cauchy-Schwarz inequality and (33) we have

$$
\begin{gathered}
\iint_{Q}\left|\int_{t-\tau(t)}^{t} c\left(u_{m}\right)(x, t, s) d s-\int_{t-\tau(t)}^{t} c(u)(x, t, s) d s\right|^{2} d x d t \leq \tau^{+} \int_{-\infty}^{0} \int_{\Omega}\left(\int_{t-\tau^{+}}^{t} \mid c\left(u_{m}\right)(x, t, s)-\right. \\
\left.\quad-\left.c(u)(x, t, s)\right|^{2} d s\right) d x d t \leq L^{2} \tau^{+} \int_{\Omega}\left(\int_{-\infty}^{0} \int_{t-\tau^{+}}^{t}\left|u_{m}(x, s)-u(x, s)\right|^{2} d s d t\right) d x \leq \\
\leq L^{2} \tau^{+} \int_{\Omega} \int_{-\infty}^{0} \int_{s}^{s+\tau^{+}}\left|u_{m}(x, s)-u(x, s)\right|^{2} d t d s d x=L^{2} \tau^{+2} \iint_{Q}\left|u_{m}(x, t)-u(x, t)\right|^{2} d t d x \underset{m \rightarrow \infty}{\longrightarrow} 0 .
\end{gathered}
$$

Thus, we obtain

$$
\int_{t-\tau(t)}^{t} c\left(u_{m}\right) d s \underset{m \rightarrow \infty}{\longrightarrow} \int_{t-\tau(t)}^{t} c(u) d s \text { strongly in } L_{2}(Q) .
$$

From condition $\left(\mathcal{A}_{2}\right)$, and estimate $(31)$ we have that the following estimate is correct

$$
\iint_{Q}\left|a_{i}\left(u_{m}\right)\right|^{p_{i}^{\prime}(x)} d x d t \leq C_{10} \iint_{Q}\left(\sum_{i=0}^{n}\left|\partial_{i} u_{m}\right|^{p_{i}(x)}+\left|h_{i}\right|^{p_{i}^{\prime}(x)}\right) d x d t \leq C_{11},
$$

where $C_{10}$ and $C_{11}$ are positive constants independent of $m$.

Hence, from (35) we obtain that $\left\{a_{i}\left(u_{m}\right)\right\}_{m=1}^{\infty}$ is bounded in $L_{p_{i}^{\prime}(\cdot)}(Q)$ for each $m \in$ $\{0, \ldots, n\}$. This and (33) yield that there exists a subsequence of $\left\{u_{m}\right\}_{m=1}^{\infty}$ (denoted also by $\left.\left\{u_{m}\right\}_{m=1}^{\infty}\right)$ and functions $\chi_{i} \in L_{p_{i}(\cdot)}(Q)(i=\overline{0, n})$ such that

$$
\begin{gathered}
\partial_{i} u_{m} \underset{m \rightarrow \infty}{\longrightarrow} \partial_{i} u, \quad \text { a.e. on } \quad Q, \quad i=\overline{0, n}, \\
a_{i}\left(u_{m}\right) \underset{m \rightarrow \infty}{\longrightarrow} \chi_{i} \quad \text { weakly in } \quad L_{p_{i}(\cdot)}(Q), \quad i=\overline{0, n} .
\end{gathered}
$$


Condition $\left(\mathcal{A}_{1}\right)$ and $(36)$ yield $a_{i}\left(u_{m}\right) \underset{m \rightarrow \infty}{\longrightarrow} a_{i}(u)$ a.e. on $\mathrm{Q}, i=\overline{0, n}$. From this relation and (37), according to [30, Lemma 1.3], it follows that

$$
a_{i}\left(u_{m}\right) \underset{m \rightarrow \infty}{\longrightarrow} a_{i}(u) \quad \text { weakly in } \quad L_{p_{i}(\cdot)}(Q), \quad i=\overline{0, n} .
$$

Let us show that the function $u$ is a weak solution of problem (6), (7). For this purpose, we let $m \rightarrow \infty$ in identity (23), taking into account (33), (38), (34) and the definition of the functions $f_{i, m}(i=\overline{0, n})$. As a result we obtain identity (11). Hence, we have proven that $u$ is a weak solution of problem (6), (7).

Third step (correctness of the estimate). From (31) and (33) it follows that estimate (13) is valid. Hence, we have proved Theorem 1.

\section{REFERENCES}

1. Alkhutov Y., Antontsev S., Zhikov V., Parabolic equations with variable order of nonlinearity, Collection of works of Institute of Mathematics NAS of Ukraine, 6 (2009), 23-50.

2. Antontsev S., Shmarev S., Extinction of solutions of parabolic equations with variable anisotropic nonlinearities, Proceedings of the Steklov Institute of Mathematics, 261 (2008), 11-21.

3. Antontsev S., Shmarev S., Evolution PDEs with nonstandard growth conditions. Existence, uniqueness, localization, blow-up, Atlantis Studies in Differential Equations, 4, Atlantis Press, Paris, 2015, xviii +409 p.

4. Bátkai A., Piazzera S., Semigroups for delay equations, in Resarch Notes in Mathematics, 10, A.K. Peters: Wellesley MA, 2005.

5. Bátkai A., Schnaubelt R., Asymptotic behaviour of parabolic problems with delays in the highest order derivatives, Semigroup Forum, 69 (2004), №3, 369-399.

6. Bokalo M.M., The unique solvability of a problem without initial conditions for linear and nonlinear elliptic-parabolic equations, Ukr. Mat. Visn., 8 (2011), №1, 55-86, (in Ukrainian); Engl. Transl.: Journ. of Math. Sciences, 178 (2011), №1, 41-64.

7. Bokalo M., Dynamical problems without initial conditions for elliptic-parabolic equations in spatial unbounded domains, Electronic Journal of Differential Equations, 178 (2010), 1-24.

8. Bokalo N.M., Problem without initial conditions for some classes of nonlinear parabolic equations, J. Sov. Math., 51 (1990), №3, 2291-2322.

9. Bokalo M.M., Buhrii O.M., Mashiyev R.A., Unique solvability of initial boundary value problems for anisotropic elliptic-parabolic equations with variable exponents of nonlinearity, Journal of nonlinear evolution equations and applications, 2013 (2014), №6, 67-87.

10. Bokalo M., Dmytriv V., On a Fourier problem for coupled evolution system of equations with integral time delays, Visnyk of the Lviv Univ. Series Mech. Math., 60 (2002), 32-49.

11. Bokalo M., Ilnytska O., Problems for parabolic equations with variable exponents of nonlinearity and time delay, Applicable Analysis, 2016, http://dx.doi.org/10.1080/00036811.2016.1183771.

12. Bokalo M., Lorenzi A., Linear evolution first-order problems without initial conditions, Milan Journal of Mathematics. 77 (2009), 437-494.

13. Bokalo M.M., Pauchok I.B., On the well-posedness of the Fourier problem for higher-order nonlinear parabolic equations with variable exponents of nonlinearity, Mat. Stud., 26 (2006), №1, 25-48.

14. Bokalo M.M., Sikorskyy V.M., About properties of solutions of problem without initial conditions for equations generalized politropic filtration equation, Visnyk of the Lviv University. Serija meh.-mat., 51 (1998), 85-98.

15. Chueshov I., Rezounenko A., Finite-dimensional global attractors for parabolic nonlinear equations with state-dependent delay, Commun. Pure Appl. Anal., 14 (2015), 1685-1704.

16. Coddington E.A., Levinson N., Theory of ordinary differential equations, New York, Toronto, London: McGraw-Hill book company, 1955. 
17. Di Blasio G., Kunisch K., Sinestrari E., $L^{2}$-regularity for parabolic partial integrodifferential equations with delay in the highest-order derivatives, Journal of Mathematical Analysis and Applications, 102 (1984), 38-57.

18. Diening L., Harjulehto P., Hästö P., Rưžička M., Lebesgue and Sobolev spaces with variable exponents, Lecture Notes in Mathematics, 2017, Springer, Heidelberg, 2011, x+509 pp.

19. Dmytriv V.M., On a Fourier problem for coupled evolution system of equations with time delays, Mat. Stud., 16 (2001), 141-156.

20. Elsholts L.E., Norkin S.B., Introduction to the theory of differential equations with deviating argument, Moscow (RF), Nauka, 1971.

21. Ezzinbi K., Liu J.H., Periodic solutions of non-densely defined delay evolution equations, Journal of Applied Mathematics and Stochastic Analysis, 15 (2002), №2, 105-114.

22. Fan X., Zhao D., On the space $L^{p(x)}(\Omega)$ and $W^{m, p(x)}(\Omega)$, Journal of Mathematical Analysis and Applications, 263 (2001), 424-446.

23. Fu Y., Pan N., Existence of solutions for nonlinear parabolic problem with p $(x)$-growth, Journal of Mathematical Analysis and Applications, 362 (2010), 313-326.

24. Jin Ch., Yin J., Traveling wavefronts for a time delayed non-Newtonian filtration equation, Physica D., 241 (2012), 1789-1803.

25. Khusainov D., Pokojovy M., Racke R., Strong and mild extrapolated $L^{2}$-solutions to the heat equation with constant delay, SIAM Journal on Mathematical Analysis, 47 (2015), №1, 427-454.

26. Kováčik O., Rákosníc J., On spaces $L^{p(x)}$ and $W^{k, p(x)}$, Czechoslovak Mathematical Journal, 41 (1991), №116, 592-618.

27. Mashiyev R.A., Buhrii O.M. Existence of solutions of the parabolic variational inequality with variable exponent of nonlinearity, Journal of Mathematical Analysis and Applications, 377 (2011), 450-463.

28. Musielak J., Orlicz spaces and modular spaces. Lecture Notes in Mathematics, 1034, Berlin-Heidelberg, Springer Verlag, 1983.

29. Mihailescu M., Radulescu V., Tersian S., Homoclinic solutions of difference equations with variable exponents, Topological Methods in Nonlinear Analysis, 38 (2011), 277-289.

30. Lions J.-L., Quelques méthodes de résolution des problémes aux limites non linéaires. Paris (France), Dunod Gauthier-Villars, 1969.

31. Oleinik O.A., Iosifjan G.A., Analog of Saint-Venant's principle and uniqueness of solutions of the boundary problems in unbounded domain for parabolic equations, Usp. Mat. Nauk., 31 (1976), №6, $142-166$.

32. Orlicz W., Uber konjugierte Exponentenfolgen, Studia Mathematica (Lwów), 3 (1931), $200-211$.

33. Pankov A.A., Bounded and almost periodic solutions of nonlinear operator differential equations, Kluwer, Dordrecht, 1990.

34. Rezounenko A.V., Wu J. A non-local PDE model for population dynamics with state-selective delay: Local theory and global attractors, Journal of Computational and Applied Mathematics, 190 (2006), 99-113.

35. Rǔžička M., Electrorheological fluids: modeling and mathematical theory. Lecture Notes in Mathematics, 1748, Berlin, Springer-Verlag, 2000.

36. Tihonov A.N., Uniqueness theorems for the heat equation, Matem. Sbornik, 2 (1935), 510-516.

37. Showalter R.E., Singular nonlinear evolution equations, Rocky Mountain J. Math., 10 (1980), №3, 499507.

38. Showalter R.E., Monotone operators in Banach space and nonlinear partial differential equations, Amer. Math. Soc., Providence, 1997, 49.

Ivan Franko National University of Lviv

mm.bokalo@gmail.com

ol.ilnytska@gmail.com 